\title{
Blast loading of concrete pipes using spherical centrically placed C-4 charges
}

\author{
Martin Kristoffersen ${ }^{1, *}$, Knut Ove Hauge ${ }^{2}$, Georgios Valsamos ${ }^{3}$, and Tore Børvik ${ }^{1,2}$ \\ ${ }^{1}$ Department of Structural Engineering, Structural Impact Laboratory (SIMLab), Norwegian University of Science and Technology \\ (NTNU), NO-7491 Trondheim, Norway \\ ${ }^{2}$ Norwegian Defence Estates Agency (NDEA), NO-0103 Oslo, Norway \\ ${ }^{3}$ European Commission, Joint Research Centre (JRC), Directorate for Space Security and Migration, Safety and Security of Buildings \\ Unit, 21027 Ispra, Italy
}

\begin{abstract}
The Norwegian Public Roads Administration has initiated a large research project on a potential ferry-free coastal highway route E39 from Trondheim to Kristiansand. Parts of this route involve crossing some wide and deep fjords, where one of the suggested crossing alternatives is a Submerged Floating Tunnel (SFT) built in concrete. A major concern regarding an SFT is internal blast loading from accidents involving gas or fuel tankers, or from terrorist attacks. To assess the performance of tubular concrete structures exposed to blast loading, $1760 \mathrm{~mm}$ long plain concrete pipes with an internal diameter of $200 \mathrm{~mm}$ and pipe wall thickness of $41 \mathrm{~mm}$ were loaded by spherical C-4 charges of various sizes up to $25 \mathrm{~g}$. Pressure sensors in different directions equidistant from the charge location recorded the blast pressure, while two high-speed cameras captured the events. Using mass produced commercial off-the-shelf pipes ensures consistent geometry and material properties. A wooden pallet was used as support for the pipes, and an electrically initiated blasting cap was used to detonate the C-4. A charge size of at least $13 \mathrm{~g} \mathrm{C}-4$ was needed to breach the pipe wall. Larger charge sizes caused longitudinal cracks and broke the pipe into oblong pieces. The test results were used for comparison with simulations using the explicit finite element solver EUROPLEXUS, intended for fast transient dynamics involving fluid-structure interaction. Both purely Lagrangian simulations and fully coupled fluid-structure interaction simulations were carried out. While the former method is more efficient computationally, the latter approach accounts for reflections and the confinement of the pipe. It can be difficult to predict the exact charge size where through-thickness cracking occurs, but the qualitative results were encouraging for further work. When accurate simulations of the experiments are obtained, the simulations will be extended to a full cross-section of a submerged floating tunnel.
\end{abstract}

\section{Introduction}

The Norwegian Public Roads Administration (NPRA) conducts a large research project aimed at making the coastal highway route E39 from Trondheim to Kristiansand ferry free [1]. The project involves crossing wide and deep fjords, where one of the suggested alternatives for crossing is a submerged floating tunnel (SFT) built in concrete. A feasibility study has concluded that this is a viable concept [2]. The concept involves various challenges; fluid-structure interaction (FSI) was studied by Remseth et al. [3] for this type of structure, while impact from ships has been investigated as well [4]. Blast loading in an SFT is currently under investigation by means of concrete slabs [5], because a major concern regarding an SFT is internal blast loading. Such loading may originate from accidents involving tankers transporting dangerous cargo like liquid natural gas or gasoline, or from a terrorist attack.

To assess the performance of tubular concrete structures exposed to blast loading, plain concrete pipes were

\footnotetext{
*e-mail: martin.kristoffersen@ntnu.no
}

loaded by centrically placed C- 4 charges of various sizes up to $25 \mathrm{~g}$. Pressure sensors in different directions equidistant from the charge location recorded the blast pressure, while high-speed cameras captured the events from two angles. Numerical simulations using EUROPLEXUS [6] have been carried out using both Lagrangian and fully coupled FSI approaches. While the onset of fracture and fragment sizes are very difficult to predict quantitatively, the numerical results showed good qualitative results.

\section{Concrete pipes}

The test specimens are commercially available off-theshelf plain concrete pipes originally intended for drainage. Reinforced pipes are outside the scope for the current study. As these plain concrete pipes are mass produced, the geometric and material properties are very similar between the specimens. The producer routinely samples the concrete and conducts cube compression tests at different stages of the curing process. Samples taken 1 day, 7 days and 28 days after casting gave a cube compressive strength $f_{c}$ of 38.2 MPa, 64.1 MPa and 83.3 MPa, respectively. The 


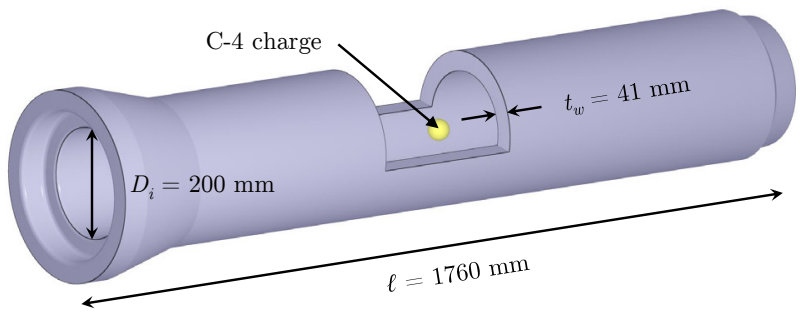

Fig. 1. Geometry of concrete pipes used in experiments.

latter value was used as input in the numerical simulations. The water/cement ratio was 0.37 , and the maximum aggregate size was $8 \mathrm{~mm}$. An estimate for the mass density was made to $3000 \mathrm{~kg} / \mathrm{m}^{3}$, reflecting the dense nature of this concrete.

A sketch of the pipe geometry is shown in Fig. 1, where the pipe is cut to show the charge position. The total length $\ell$ of each pipe is $1760 \mathrm{~mm}$, and the internal diameter $D_{i}$ is $200 \mathrm{~mm}$. The pipe wall thickness $t_{w}$ is $41 \mathrm{~mm}$, making the outer dimater $D_{o}=282 \mathrm{~mm}$. At the pipe ends, the geometry is made so that it is possible to join the pipes to make a long, continuous drainage pipeline.

\section{Blast testing}

\subsection{Experimental setup}

A picture of the experimental setup is shown in Fig. 2. When testing, the concrete pipes rest on a wooden pallet which provides continuous support and prevents them from rolling. As shown in Fig. 1, the C-4 charges used in this study are spherical and placed in the centre of each concrete pipe specimen. The charge was placed on a styrofoam support, and an electrically ignited blasting cap (approximately $1 \mathrm{~g} \mathrm{C}-4$ equivalent) was used to initiate the blast.

Three Kistler 603B piezoelectric pressure sensors with a sampling frequency of $1 \mathrm{MHz}$ were used to record the pressure at equidistant points approximately $1315 \mathrm{~mm}$ from the charge - one in each direction along the pipe's longitudinal axis (sensors 1 and 3 in Fig. 2), and one perpendicular to the main axis (sensor 2 in Fig. 2). A steel rod through the concrete pipes was used to align the pressure sensors. Each pressure sensor was flush-mounted on

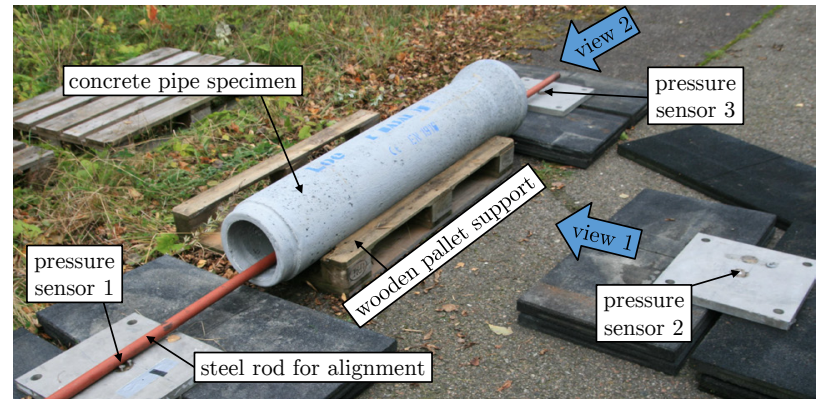

Fig. 2. Photograph of the experimental setup.
Table 1. Experimental matrix sorted by charge size $W$.

\begin{tabular}{rccccl}
\hline ID & $\begin{array}{c}R \\
{[\mathrm{~mm}]}\end{array}$ & $\begin{array}{c}W \\
{[\mathrm{~g}]}\end{array}$ & $\begin{array}{c}Z \\
{\left[\mathrm{~m} \cdot \mathrm{kg}^{-1 / 3}\right]}\end{array}$ & $\begin{array}{c}P_{\max } \\
{[\mathrm{bar}]}\end{array}$ & Comment \\
\hline III & 100 & 10.0 & 0.464 & 2.22 & intact \\
IV & 100 & 12.5 & 0.431 & 2.64 & surf. crack \\
XIV & 100 & 13.0 & 0.425 & 2.40 & breach \\
VI & 100 & 13.5 & 0.420 & 2.69 & breach \\
V & 100 & 14.0 & 0.415 & 2.57 & breach \\
II & 100 & 15.0 & 0.405 & 2.77 & breach \\
I & 100 & 25.0 & 0.342 & 3.32 & breach \\
\hline
\end{tabular}

an aluminium plate measuring $400 \times 400 \times 30 \mathrm{~mm}$, thus keeping them firmly in place.

In addition, high-speed cameras operating at 22000 frames per second were used to capture the events. The fields of view for the cameras are indicated by arrows in Fig. 2. The cameras were placed inside steel containers for protection.

In total, seven concrete pipes were tested with charge masses $W$ ranging from $10 \mathrm{~g}$ to $25 \mathrm{~g}$. The standoff distance $R$, meaning the distance from the charge centre to the closest part of the pipe, is half the inner diameter, which is $100 \mathrm{~mm}$ for all cases. From $R$ and $W$, the scaled distance $Z$ is obtained,

$$
Z=\frac{R}{\sqrt[3]{W}}
$$

The maximum pressure $P_{\max }$ recorded among the three pressure sensors in each experiment is listed in Table 1 along with other test parameters.

\subsection{Test results}

For the smallest charge (10 g C-4 for pipe III), no cracks were noted in the pipe. The pressure recordings by sensors 1 and 3 from this test are shown in Fig. 3. Results from sensor 2 are omitted, because it was difficult to extract anything useful. Increasing the charge size to $12.5 \mathrm{~g}$ for pipe IV produced long longitudinal surface cracks along the pipe, but no through-thickness cracks were noted and the pipe maintained its integrity and remained in one piece.

The smallest charge managing to crack the pipe into pieces was $13.0 \mathrm{~g}$ (pipe XIV). This caused the pipe to break into few and fairly large oblong pieces. Increasing the charge size beyond this produced more fragments but of smaller size. The end sections of the concrete pipes always remained whole, so the fragmentation was confined to the central area.

The pressure recordings were consistent with respect to charge size, although they can be difficult to distinguish when the difference in charge size is less than one gram. Reflections, local differences, charge position and sensor alignment all affect the pressure readings to some extent. In some of the tests, the fireball exiting the pipe caused some fluctuations in the pressure readings. This can be seen for sensor 1 in Fig. 3, but more revealing examples were also observed.

From the high speed videos, it was observed that oblong pieces formed through longitudinal cracking. An ex- 


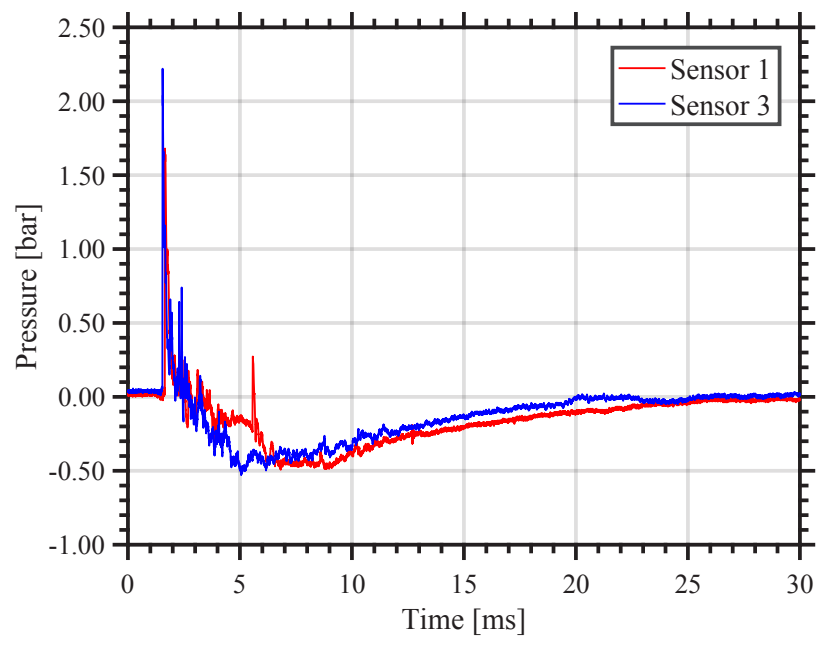

Fig. 3. Pressure-time histories from sensors 1 (red) and 3 (blue) from testing of pipe III (10 g C-4).

ample of this (pipe I) is shown Fig. 4, where a charge size of $25 \mathrm{~g}$ was used. Yellow arrows in the figure indicate some of the fragments from the pipe. $25 \mathrm{~g}$ was the largest charge size, so for the other pipes the pieces were generally larger and fewer in numbers because less energy was delivered to the pipe.

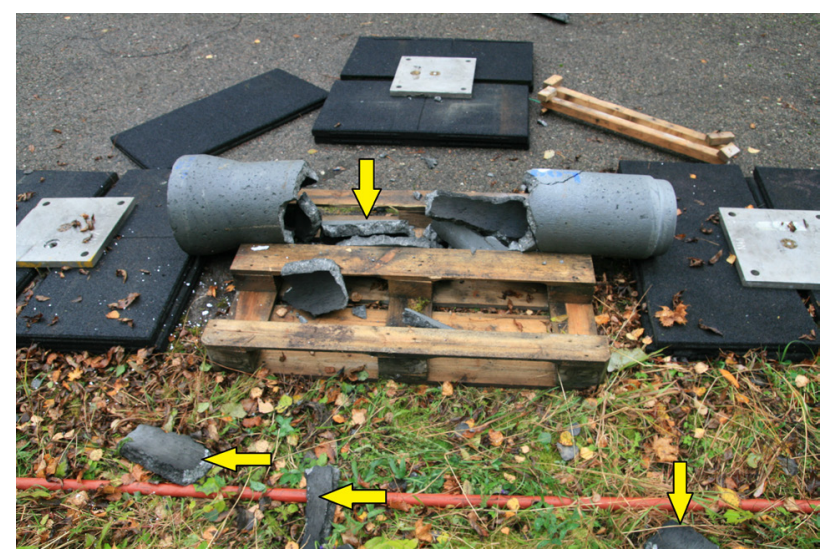

Fig. 4. Pipe I ( 25 g C-4 charge) after testing, with pieces scattered around the perimeter.

\section{Numerical simulations}

The simulation herein are carried out using the explicit finite element solver EUROPLEXUS [6] intended for fast transient dynamics. The EUROPLEXUS software offers a diverse material library and is capable of running fully coupled fluid-structure interaction simulations.

The main goals of the numerical part are to evaluate the differences between using a purely Lagrangian model and a coupled Lagrangian-Eulerian approach, and to obtain a qualitative assessment of the performance of the numerical models on blast loaded concrete pipes.

\subsection{Numerical setup}

The pipe geometry shown in Fig. 1 is discretised by 8 node linear brick elements with one integration point, and an element size of approximately $10 \mathrm{~mm}$. Mesh adaptivity based on the volumetric strain is employed for the concrete pipe. The refinement halves the element lengths when the volumetric strain reaches a magnitude of 0.010 , so one solid element is divided into eight. An element is eroded when the volumetric strain value reaches 0.030 . Other values of these parameters may give different results, so a parameter study is suggested for further work.

A three-invariant cap model with mixed hardening has been used to represent the concrete. The model is called "Dynamic plastic damage concrete" (DPDC) in EUROPLEXUS [6]. It includes strain rate sensitivity and isotropic damage, and has been used with good results in previous work [7]. Material parameters are given in Section 2.

Two different approaches have been used to describe the load - one purely Lagrangian and the other a fully coupled FSI approach (see Fig. 5). The first, based on experimental data obtained by Kingery and Bulmash [8], consists of applying a pressure-time history to the inner surface of the concrete pipe based on the charge size, the distance to the charge, and the angle between each element's surface normal and the direction towards the charge center. The Friedlander equation describes the pressure-time history. The other approach consists of modelling the charge by using the Jones-Wilkins-Lee (JWL) equation of state with suitable parameters [9], and the fluid medium (air) through which the blast wave propagates. The fluid was discretised by 8-node cell-centered finite volumes, with length $10.0 \mathrm{~mm}$. The finite volume size was refined to $2.5 \mathrm{~mm}$ in the vicinity of the charge.

In the FSI simulations, the fluxes in the fluid mesh are blocked across the fluid-structure interface, thereby transferring the pressure from the detonation to the structure. The structure then responds and deforms according to the load. This alters the fluid-structure interface, and completes the feedback loop between the fluid and the structure. In addition, a reflective boundary has been placed on the same level as the ground to account for reflections. The remaining boundaries were absorbing boundaries, allowing the pressure to exit the fluid domain freely if necessary.

\subsection{Simulations results}

The simulations generally show good qualitative results. Cracks appear in the longitudinal direction for both the FSI calculations and the Lagrangian calculations as shown in Fig. 5 for a charge size of 40 grams. This charge size is used to amplify the differences between the Lagrangian and FSI approaches. In the Lagrangian simulation, the strain in the pipe is distributed across a smaller area compared with the FSI case. It is observed that the FSI approach resembles the experiments more closely. Pieces of similar shape as in the tests detach from the pipe, depicted 


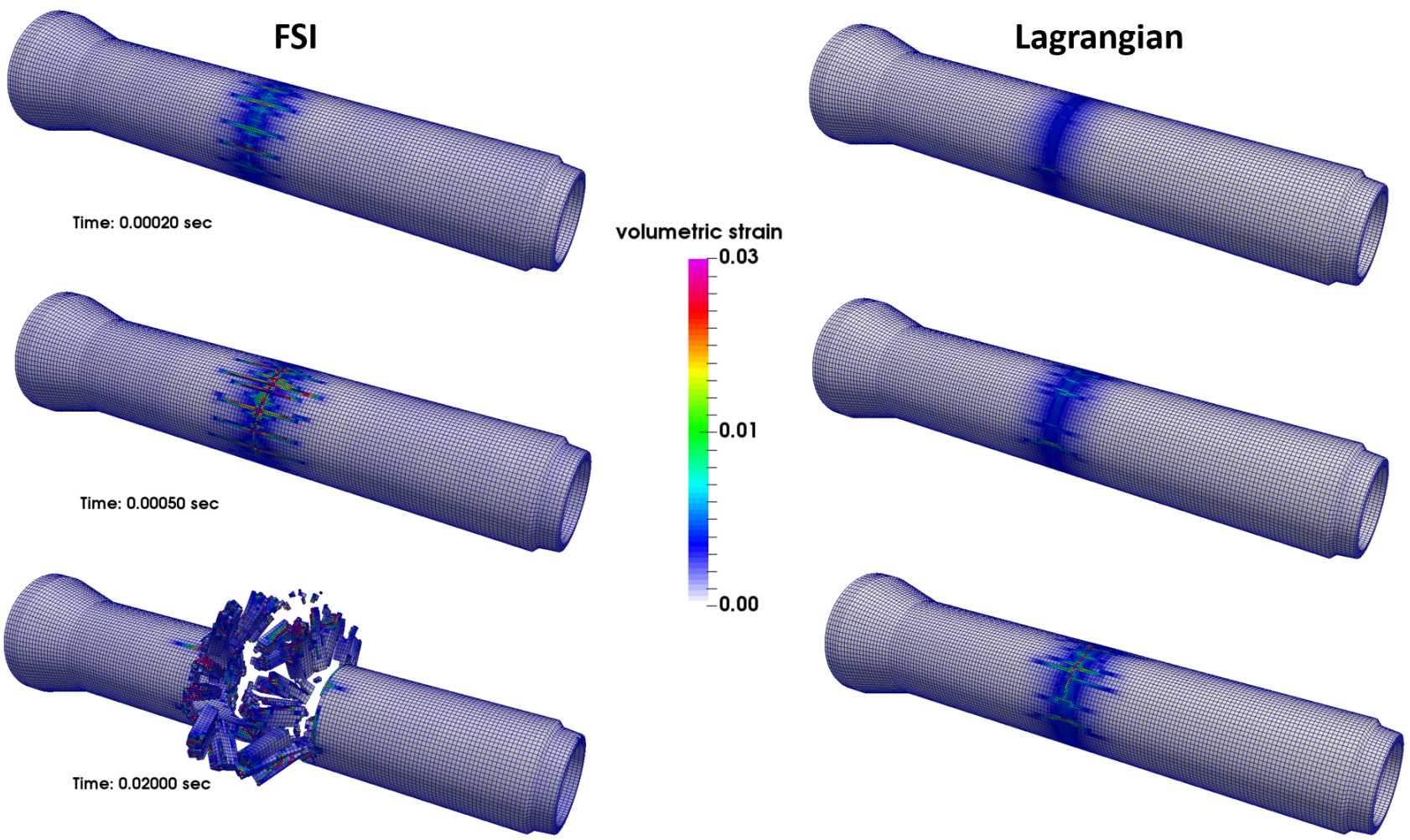

Fig. 5. A contour plot of the volumetric strain in in the concrete pipe is shown of a fully coupled FSI simulation (left) and for a Lagrangian simulation (right) at three different points in time for a charge size of $40 \mathrm{~g} \mathrm{C}-4$.

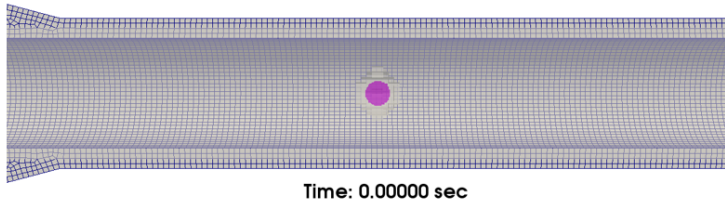

Time: $0.00000 \mathrm{sec}$

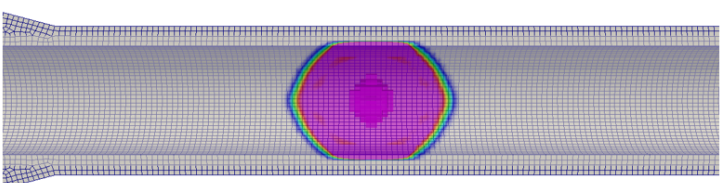

Time: $0.00003 \mathrm{sec}$

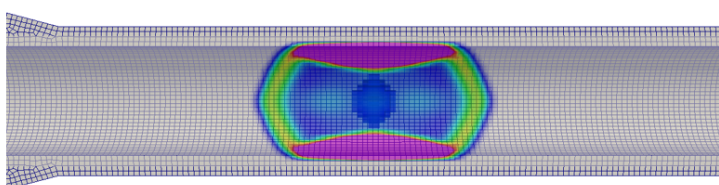

Time: $0.00005 \mathrm{sec}$

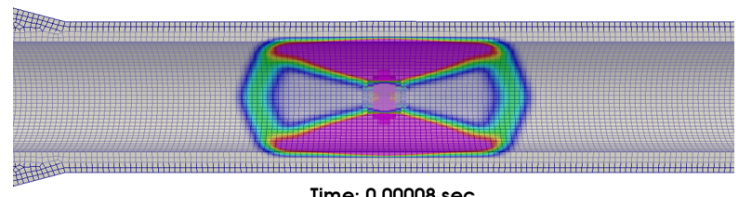

Time: $0.00008 \mathrm{sec}$
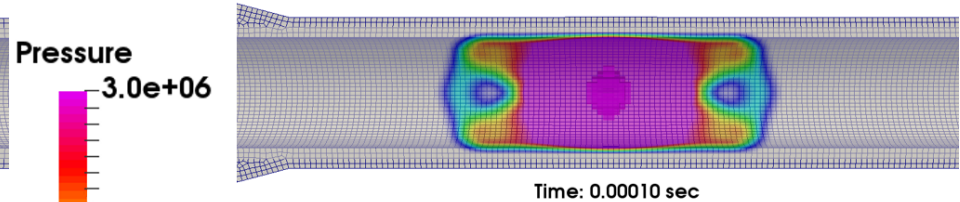

Time: $0.00010 \mathrm{sec}$

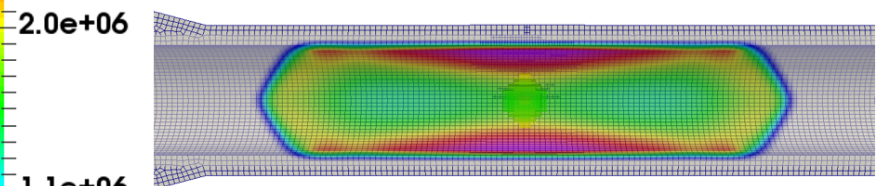

$1.1 e+06$

Time: $0.00020 \mathrm{sec}$

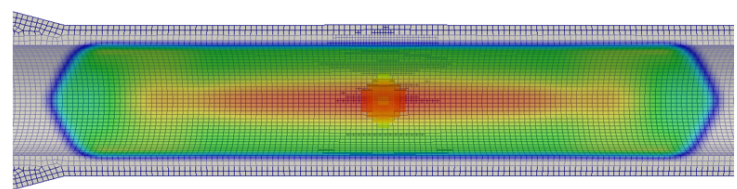

Time: $0.00028 \mathrm{sec}$

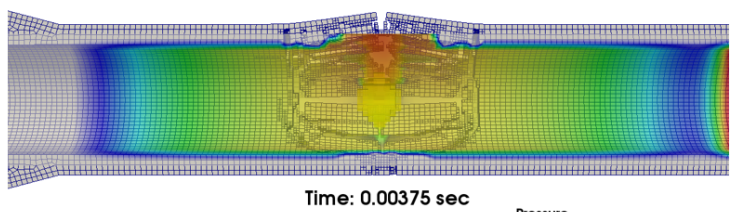

$1.00+05$ $1.1 e^{+05} \quad 1.2 e+05$

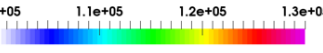

Fig. 6. The fringe plot shows the pressure wave propagation including reflections in the fluid inside the pipe for a fully coupled FSI simulation (40 g C-4). Note that the small legend in the final frame (bottom right) has been scaled down to highlight the pressure distribution. 


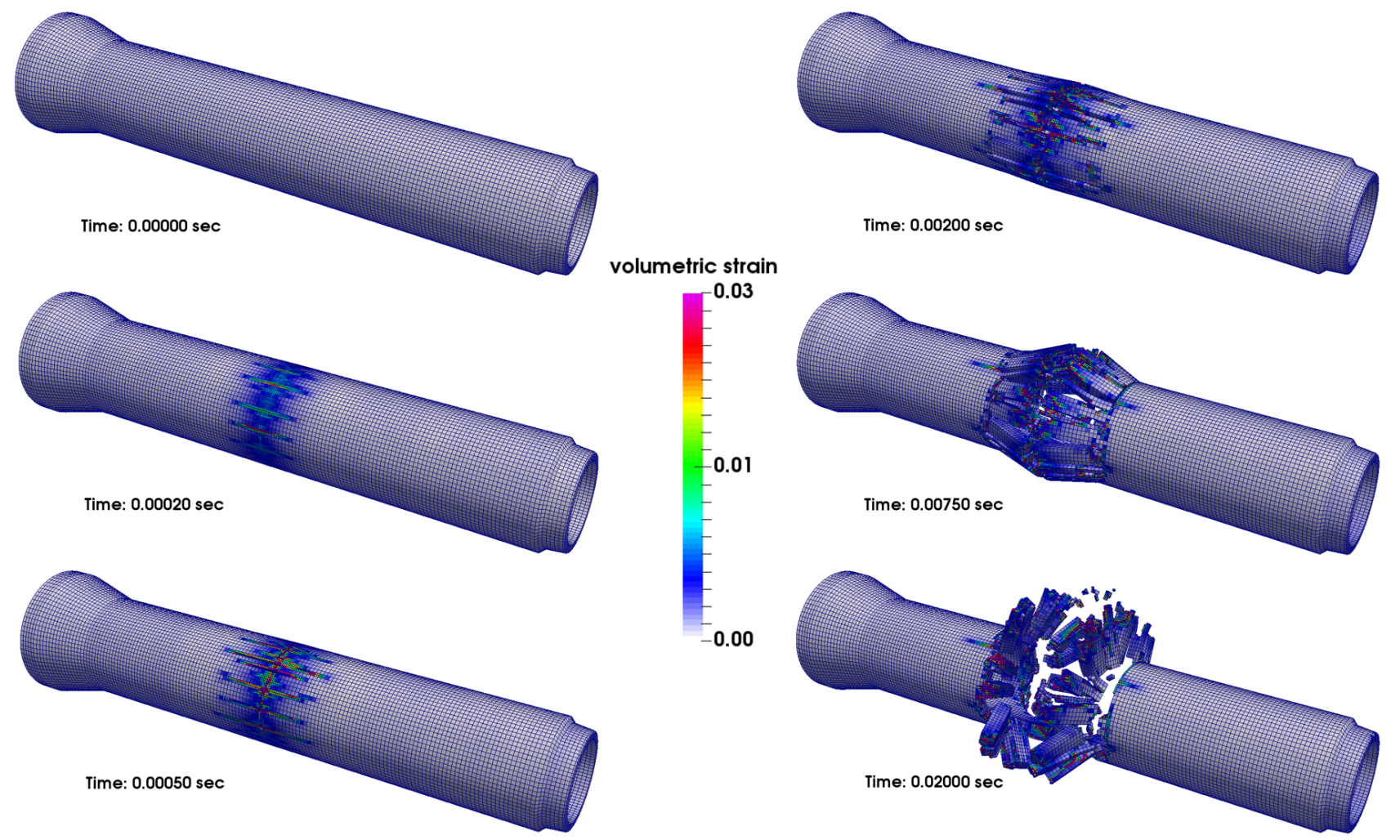

Fig. 7. The volumetric strain in the concrete pipe from an FSI simulation is shown at different points in time for $40 \mathrm{~g} \mathrm{C}-4$.

in the lower left part Fig. 5. This result comes from the fact that FSI is able to account for the confinement and reflections inside the pipe, while the Lagrangian technique only applies a predetermined pressure-time history to the pipe surface. This point is further emphasised by Fig. 6, which shows the pressure distribution on the inside of the pipe during an FSI calculation. At time $t=0.00010 \mathrm{~s}$, the fluid pressure is high, and at $t=0.00020 \mathrm{~s}$ the mesh refinement has initiated in the pipe. The pressure propagates down the pipe and causes large hoop stresses in the pipe, amplified by reflections in the fluid domain. When the pipe wall finally cracks, the pressure is free to escape. Note that the final frame (lower right) has a scaled down legend to highlight the pressure distribution.

Fig. 7 shows the concrete pipe at various stages during the FSI simulation. Cracks appear in the same manner as in the experiments, and the crack propagation is along the axial direction of the pipe. The mesh adaptivity appears to work well (this also applies to the Lagrangian case), clearly evident at $t=0.00050 \mathrm{~s}$. At $t=0.00750 \mathrm{~s}$, oblong fragments are forming and through-thickness cracks allow the pressure to escape and tear the pipe into fragments. In the final frame, pieces are flying off with the two pipe ends remaining fairly intact. Qualitatively, this is close to the experimental result, an example of which can be seen in Fig. 4. The Lagrangian case does not capture this fragmentation satisfactorily.

While the coupled analyses are superior in accuracy and in including the physical phenomena at play, Lagrangian analyses can still be useful for making initial estimates before a more accurate approach is adopted.
Based on Fig. 5, Lagrangian analyses appear to give nonconservative results. This means that Lagrangian results of a full SFT should be used with care. Naturally, the FSI simulations require more CPU time. On average, the Lagrangian calculations take roughly 42 minutes per millisecond of simulation time. This increases to approximately $213 \mathrm{~min} / \mathrm{ms}$ for the FSI approach, thus increasing the simulation time by a factor of 5 .

\section{Concluding remarks}

The experimental campaign was able to determine the minimum charge size able to breach the concrete pipe wall. When the pipe broke, oblong pieces formed with the longitudinal cracking of the pipes as depicted in Fig. 8. Increasing the charge mass resulted in more fragments of smaller size. The end sections of the pipe generally remained intact, indicating that this is a local problem. After a certain distance the blast wave becomes one-dimensional along the pipe axis. This is helpful to remember when moving on to simulations of a full SFT cross-section in the future: only the part closest to the blast need to be modelled in detail.

Pressure sensors registered the blast wave escaping the pipe, and the recordings were consistent among the different tests. As the difference between the charge sizes were as little as $0.5 \mathrm{~g}$, some of the pressure-time curves were difficult to discern. The pressure recordings were characteristic of a blast load, although the fireball from the burning caused some fluctuations. 


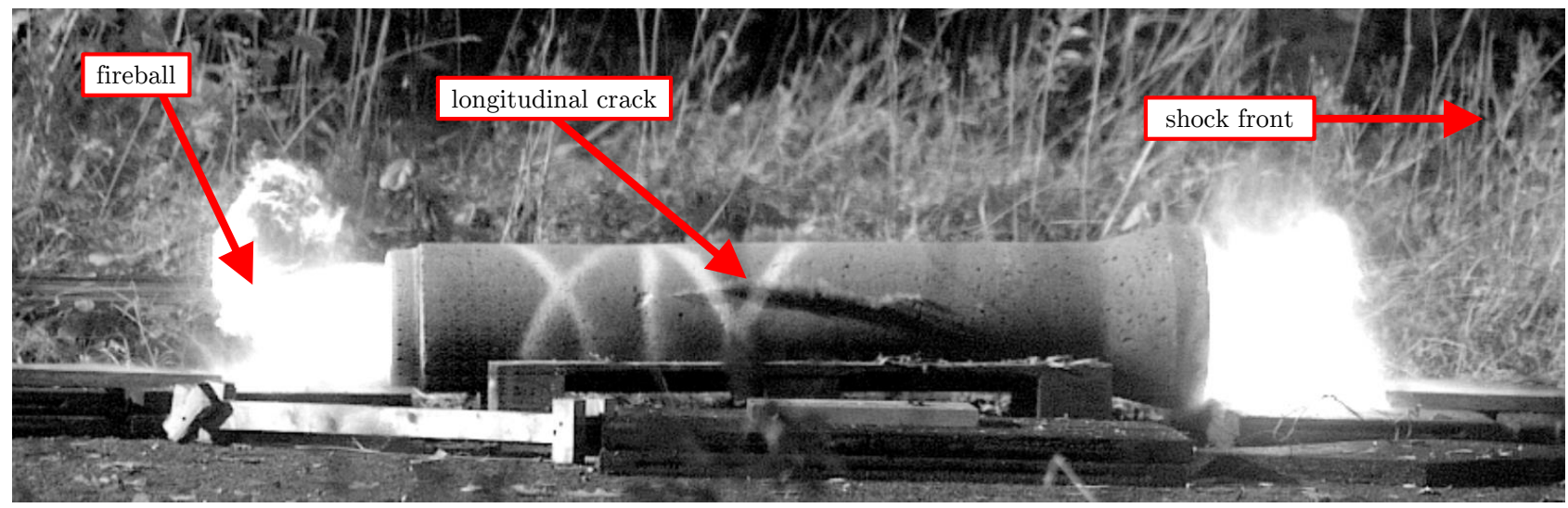

Fig. 8. Image from high-speed camera shown the longitudinal cracking of pipe XIV. The shock front can also be observed (easier with moving pictures).

Without any in-depth approach to obtaining detailed material parameters, and without any specific tuning of the simulation parameters, both the Lagrangian and the coupled simulations gave good qualitative results, but the coupled simulations were closer to the experimental results. Lagrangian results can still be useful, but the results should be used with a caveat because they are nonconservative. The FSI approach was superior in accuracy, in that it was able to account for the confinement and reflections in the pipe. Further, the fragmentation of the pipe obtained with FSI simulations resembled the experiments closely. Adaptivity of the structural mesh helped describe the crack propagation.

The next step of the numerical work is to improve the quantitative results. A modification of the material parameters and the parameters in JWL model could help improve the predictive accuracy of the simulations. Reflections from the ground and from the support plates for the pressure sensors are not entirely accounted for. For this reason, getting the pressure recordings from the FSI simulations to match the experimental data is left for further work. Finally, extending the simulations to a full SFT cross-section is a natural progression from the current work.

The authors would like to express their gratitude to the Norwegian Public Roads Administration and the E39 Coastal Highway Route project for funding this study. Additional thanks to the Norwegian Defence Estates Agency for providing the test range and equipment necessary for conducting the experiments.

\section{References}

1. Statens Vegvesen (Accessed 05.01.2018), http://www. vegvesen.no/Vegprosjekter/ferjefriE39/English

2. A. Fjeld, T. Søreide, S. Haugerud, Tech. rep., Reinertsen AS, Dr. Techn. Olav Olsen (2013), document no. 11744-ROO-R-002

3. S. Remseth, B. Leira, K. Okstad, K. Mathisen, T. Haukås, Computers and Structures 72, 659 (1999)

4. Y. Xiang, Y. Yang, Marine Structures 53, 20 (2017)

5. M. Kristoffersen, J. Pettersen, V. Aune, T. Børvik, Submitted for possible journal publication (under review). (2018)

6. Europlexus user's manual, Joint Research Centre (Cited 16.12.2017), http://europlexus.jrc.ec.europa.eu/ public/manual $\_$pdf/manual.pdf

7. D. Guilbaud, XIII International Conference on Computational Plasticity, Barcelona, Spain pp. 1031-1042 (2015)

8. C. Kingery, G. Bulmash, Tech. rep., Aberdeen Proving Ground, MD: Defence Technical Information Center, Ballsitic Research Laboratory (1984)

9. B. Dobratz, P. Crawford, LLNL Explosives HandbookProperties of chemical explosives and explosive simulants, Lawrence Livermore National Laboratory (1985) 